\title{
Design of a High Speed XAUI Based on Dynamic Reconfigurable
}

\section{Transceiver IP Core}

\author{
${ }^{*}{ }_{1}$ Haipeng Zhang, ${ }^{1}$ Lingjun Kong, ${ }^{2}$ Xiuju Huang, ${ }^{3}$ Mengmeng Cao \\ 1. School of Electronics \& Information, Hangzhou Dianzi University, Hangzhou, China, \\ 310018 \\ 2. UTSTARCOM Co. Ltd. Hangzhou, China, 310052 \\ 3. North China Electric Power University, Department of electronics and Communication \\ Engineering, Baoding, China, 071003 \\ Email:1islotus@163.com, ${ }^{2}$ xjhuang@utstar.com, ${ }^{3}$ caomengmeng520@126.com
}

Abstract. By using the dynamic reconfigurable transceiver in high speed interface design, designer can solve critical technology problems such as ensuring signal integrity conveniently, with lower error binary rate. In this paper, we designed a high speed XAUI (10Gbps Ethernet Attachment Unit Interface) to transparently extend the physical reach of the XGMII. The following points are focused: (1) IP (Intellectual Property) core usage. Altera Co. offers two transceiver IP cores in Quartus II MegaWizard Plug-In Manager for XAUI design which is featured of dynamic reconfiguration performance, that is, ALTGX_RECONFIG instance and ALTGX instance, we can get various groups by changing settings of the devices without power off. These two blocks can accomplish function of PCS (Physical Coding Sub-layer) and PMA (Physical Medium Attachment), however, with higher efficiency and reliability. (2) $1+1$ protection. In our design, two ALTGX IP cores are used to work in parallel, which named XAUIO and XAUI1. The former works as the main channel while the latter redundant channel. When XAUI0 is out of service for some reasons, XAUI1 will start to work to keep the business. (3) RTL (Register Transfer Level) coding with Verilog HDL and simulation. Create the ALTGX_RECONFIG instance and ALTGX instance, enable dynamic reconfiguration in the ALTGXB Megafunction, then connect the ALTGX_RECONFIG with the ALTGX instances. After RTL coding, the design was simulated on VCS simulator. The validated result indicates that the packets are transferred efficiently. FPGA makes high-speed optical communication system design simplified.

\section{Keywords: High speed XAUI, Dynamic reconfigurable transceiver, EBR, FPGA}

* Corresponding Author:

Haipeng Zhang

School of Electronics \& Information, Hangzhou Dianzi University,

Hangzhou, China, 310018

Email: islotus@163.com 


\section{Introduction}

With the development of Internet, the need for higher bandwidth and data rate is driven largely by the demand to upgrade existing communication systems and the emergence of new applications. Communication equipment on market has the trend of lower power and lower cost, higher speed transportation with a more compact size.

The next generation of IC product uses the 45-nm or 40-nm process [1], even the 28-nm process to integrate more functions, so that higher operating performance and higher logic density can be achieved. However, the key to meet the increasing demand for bandwidth is that we use more and faster high-speed serial transceivers. Data links with transceivers support higher data throughput, more power efficient and higher system integration. By using transceiver technology, designers can solve critical technology issues that exist in high speed data link designs, such as Signal Integrity, Power Consumption and Board Complexity.

Altera Corporation announced the availability of a $10 \mathrm{G}$ Ethernet $(10 \mathrm{GbE})$ reference design targeting designers using the XAUI communications protocol [2], in the aims at addressing the demands of broadband networking and telecommunication applications. Altera offers a broad portfolio of devices with embedded transceivers that support dynamic reconfiguration, both FPGAs and ASICs (Application Specific Integrated Circuit). These are the Arria II GX FPGA family, the Stratix IV GX and GT FPGA families and the HardCopy IV GX ASIC family. They provide a dedicated mode for implementing the XAUI interface and allow the integration of multiple PHYs (Physical) and MACs (Media Access Control) into a single FPGA. The solution enables simple, fast protocol implementation, which reduces design risk, shortens development times, and allows you to concentrate on the core functions of the system design.

\section{Dynamic reconfigurable architecture}

Dynamically reconfiguration means reconfigurable device of the chip can be reconfigured repeatable, and performs different functions at different times [3].So we can get various groups by changing settings of the devices without power off. Compared with static reconfiguration, dynamic reconfiguration can use the reconfigurable device more thoroughly.

\subsection{Dynamic reconfigurable transceiver introduction}

Figure 1 shows the channel architecture of Arria II GX transceiver. Each transceiver channel consists of a transmitter channel and a receiver channel. Each transmitter or receiver channel is composed of one channel PCS block and one channel PMA block [4][5]. Some modules in PCS block are optional, which can be bypassed.

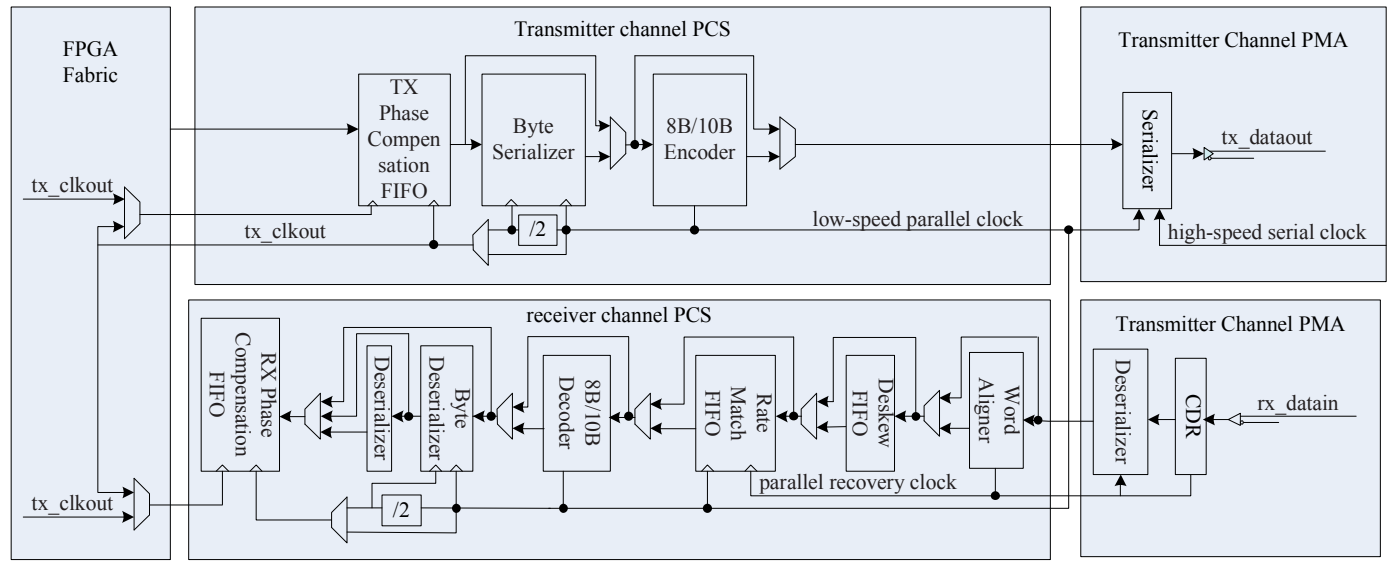

Figure 1. Transceiver channel architecture for Arria II GX

Transmitter PCS consists of the TX phase compensation FIFO, byte serializer, and 8B/10B 
encoders. While the receiver PCS consists of the word aligner, deskew FIFO, rate-match FIFO, 8B/10B decoder, byte paralyzer, byte ordering, and RX phase compensation FIFO.

Transmitter PMA consists of the serializer and the transmitter output buffer. Receiver PMA has the receiver input buffer, CDR, and paralyzer. The PMA module supports analog interface from the transceiver to medium outside.

\subsection{Dynamic reconfiguration mode}

There are four dynamic reconfiguration modes in Arria II GX devices: Offset Cancellation for receiver channels, Analog Control, Data rate division in transmitter (TX), Channel and TX PLL select/reconfig. Selection of these modes allows you to dynamically reconfigure various channel and clock multiplier units (CMU) settings without powering the device off [6].

Different decisions will be made, which depends on various reconfiguration requirements and application environments when we are choosing reconfiguration mode in the design. Table 1 lists the reasons to reconfigure the transceivers and the reconfiguration modes for various reconfiguration requirements.

Table 1. Reconfiguration modes for various reconfiguration requirements

\begin{tabular}{|l|l|}
\hline \multicolumn{1}{|c|}{ Reason for Reconfiguration } & \multicolumn{1}{|c|}{ Reconfiguration Mode to Use } \\
\hline $\begin{array}{l}\text { Counter offset variations due to process, voltage, and } \\
\text { temperature (PTV) for analog circuits. } \\
\text { Mandatory feature if you use receivers. }\end{array}$ & Offset Cancellation \\
\hline $\begin{array}{l}\text { Fine-tune signal integrity by adjusting the transmitter } \\
\text { and receiver buffer settings while bringing up a link }\end{array}$ & Analog Controls \\
\hline $\begin{array}{l}\text { Increase or decrease the data rate (/1,/2,/4) for auto } \\
\text { negotiation }\end{array}$ & $\begin{array}{l}\text { Data rate division in TX or } \\
\text { Channel and TX PLL select/reconfig }\end{array}$ \\
\hline $\begin{array}{l}\text { Support multiple protocols with the same transceivers } \\
\text { to add design flexibility }\end{array}$ & $\begin{array}{l}\text { Analog Controls } \\
\text { Channel and TX PLL select/reconfig }\end{array}$ \\
\hline $\begin{array}{l}\text { Reset the CMU PLLs through Channel and TX PLL } \\
\text { select/reconfig }\end{array}$ & Channel and TX PLL select/reconfig \\
\hline
\end{tabular}

\subsection{Dynamic reconfiguration architecture}

IP cores are used in this design and a technology of high-performance IP core multiplex is chosen. These IP cores must be verified to make sure that they work well. Therefore, design efficiency can be improved if higher reliability and lower design risk is achieved.

Arria II GX device offers two instances to support dynamic reconfiguration: ALTGX RECONFIG and ALTGX [7]. Figure 2 illustrates the connection relationships between them. 


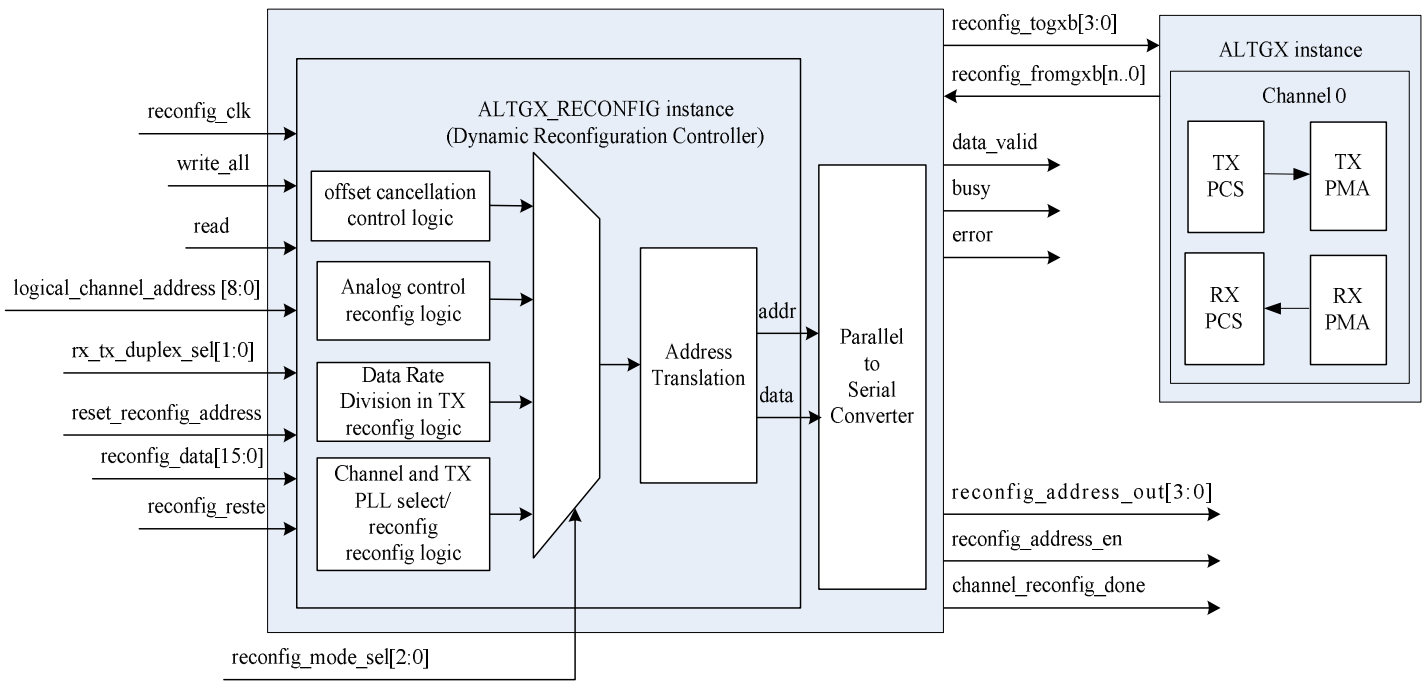

Figure 2. Connection relationships between ALTGX_RECONFIG and ALTGX

(1) ALTGX_RECONFIG instance

The ALTGX_RECONFIG instance generated by the ALTGX_RECONFIG MegaWizard Plug-In Manager represents the dynamic reconfiguration controller. It provides simple way to change transceiver PMA settings dynamically.

(2) ALTGX instance

The ALTGX instance generated by the ALTGX MegaWizard Plug-In Manager represents the transceiver. This term is used as the functional module.

\section{High speed XAUI design}

As the demand for bandwidth and transfer rate increases, high speed transceiver plays a more important role in high speed interface design. The design based on dynamic reconfigurable transceiver of FPGA can fit the bill to some extent [8]. Altera and Xilinx have already launched their own FPGA devices which meet the requirements above. With these devices, design of high speed interface becomes more simple, nevertheless, more realizable. And all this will make further promotion in the development of optical fiber communication and wireless devices.

In this part, we designed a high speed XAUI to transparently extend the physical reach of the XGMII, that is, the data output should meet XGMII style.

\subsection{XAUI brief introduction}

XAUI, the 10 Gigabit Attachment Unit Interface, is a technical innovation that dramatically improves and simplifies the routing of electrical interconnections. Developed by the IEEE 802.3ae 10 Gigabit Ethernet Task Force, XAUI delivers 10Gbps of data throughput using four differential signal pairs in each direction [9].

Generally, XAUI is designed as XGMII (10 Gigabit Medium Independent Interface) extended sublayer. The XGMII provides full duplex operation at a rate of $10 \mathrm{GbPs}$ between the MAC and PHY. Each direction is independent and contains a 32-bit data path, as well as clock and control signals. In total the interface is 74 bits wide [10]. Figure 3 shows the data stream between XGMII and XAUI: 


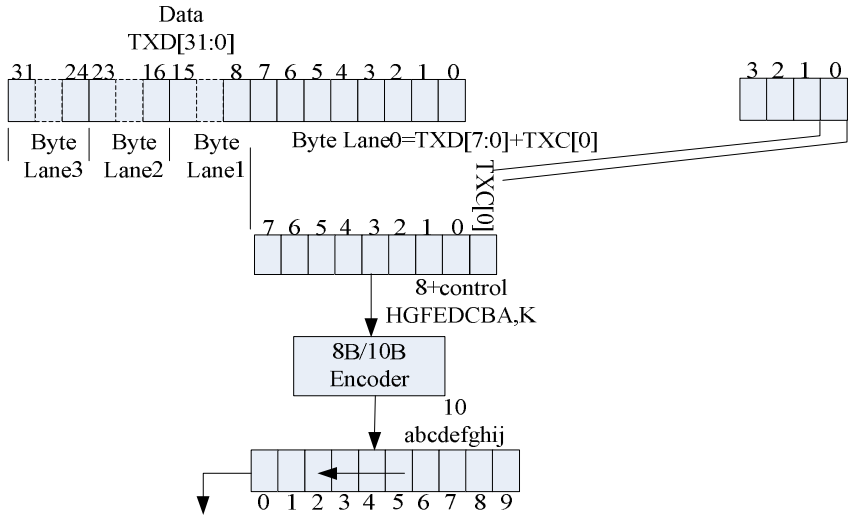

Figure 3. Data stream between XGMII and XAUI

The XGMII interface consists of four 8-bit lanes. At the transmit side of the XAUI interface, the data and control characters are converted within the XGMII extender sublayer (XGXS) into an $8 \mathrm{~B} / 10 \mathrm{~B}$ encoded data stream. Each data stream is then transmitted across a single differential pair running at $3.125 \mathrm{Gbps}$ [11]. At the XAUI receiver, the incoming data is decoded and mapped back to the 32-bit XGMII format. This provides a transparent extension of the physical reach of the XGMII and also reduces the interface pin count.

\subsection{High speed XAUI design}

The dynamic reconfigurable transceiver embedded in Arria II GX FPGA device is based on $40-\mathrm{nm}$ process. It can provide various kinds of protocols, such as PCI (Peripheral Component Interconnect) Express, serial digital interface, XAUI and so on.

When do research on application of dynamic reconfigurable transceiver in high speed XAUI, we choose the Analog Controls mode. Adjust the parameters of some options, for example, equalizer DC gain, transmit output differential voltage $\left(\mathrm{V}_{\mathrm{OD}}\right)$, pre-emphasis and setting of equalization, then we will gain the best eye diagram which means the best signal integrity. Something should be noticed that we use receiver channels in the design, so it is necessary to choose Offset Cancellation mode acquiescently. In this section, we will introduce a design proposal of high speed XAUI with dynamic reconfigurable transceiver, and Verilog HDL coding style is used [12]. Figure 4 illustrates the system top view of the design.



Figure 4. System level view of High speed XAUI interface 
XAUI LMPI module functions as the second-level microprocessor interface logic, so the related state and control registers of XAUI can be read and written. Also, we define some registers in this module.

XAUI_RECONFIG module functions as the transceiver controller instance, which is responsible for controlling the high speed XAUI channels. Options in Analog Controls mode have default settings, as the following list: tx_vodctrl is configured to 4 , tx preemp to 0, rx eqctrl to 0 , rx_eqdcgain to 0. Signal lmpi_write_all is used to start the write transportation between XAUI _EECONFIG instance and the XAUI instance, while signal lmpi_read starts the read transportation which is used in PMA control mode only.

XAUI0 and XAUI1 module act as the transceiver instance, implement basic function of high speed XAUI interface. When generate XAUI instance in Quartus II MegaWizard, we set the option "which protocol will you be using" in Parameter Settings to XAUI. Because each channel in the XAUI supports transfer rate up to $3.2 \mathrm{Gbps}$, it is necessary for us to bind 4 such channels together to achieve 10Gbps transfer rate, which means that the option "what is the number of channels" should be set to 4 .

\subsection{Signal description}

In the work of RTL coding, we should connect the ALTGX_RECONFIG instance with the ALTGX instances. The most important work is that signals must be connected to the related ports. Table 2 lists the input and output ports of the XAUI design, Table 3 lists the internal and external signals of XAUI_RECONFIG.

Table 2. Input and Output Ports of XAUI

\begin{tabular}{|l|l|l|}
\hline Signal Name & I/O Type & Description \\
\hline phy_mgmt_clk & Input & Clock signal \\
\hline rst & Input & Global asynchronous reset signal \\
\hline gmpi_xaui_cs & Input & Chip select signal \\
\hline gmpi_addr[7:0] & Input & Address signal \\
\hline gmpi_data[15:0] & Input & Input data signal \\
\hline gmpi_wen & Input & Write enable signal \\
\hline gmpi_ren & Input & Read enable signal \\
\hline xaui_sel & Input & $\begin{array}{l}\text { XAUI bus select control signal: } \\
\text { 0:XAUI0 } \\
1: X A U I 1\end{array}$ \\
\hline xaui0_xgmii_rxd[63:0] & Output & $\begin{array}{l}\text { Internal XGMII received data from XAUI0, signal } \\
\text { xaui_sel is set to 0 }\end{array}$ \\
\hline xaui0_xgmii_rxc[7:0] & Output & Internal XGMII receive control from XAUI0 \\
\hline xaui1_xgmii_rxd[63:0] & Output & Internal XGMII received data from XAUI1 \\
\hline xaui1_xgmii_rxc[7:0] & Output & Internal XGMII receive control from XAUI1 \\
\hline
\end{tabular}

Table 3. Internal and External Signals of Dynamic Reconfiguration Controller

\begin{tabular}{|l|l|l|}
\hline Signal Name & I/O Type & Description \\
\hline lmpi_rx_eqdegain & Input & Equalizer DC gain write control. \\
& & Legal settings allowed for this signal: \\
& 2 'b00 $=>0 \mathrm{~dB}$ \\
& 2 'b01 $=>3 \mathrm{~dB}$ \\
& 2 'b10 $=>6 \mathrm{~dB}$ \\
& All other values $=>$ N/A \\
\hline
\end{tabular}




\begin{tabular}{|c|c|c|}
\hline lmpi_rx_eqctrl & Input & $\begin{array}{l}\text { Write control to write an equalization control value } \\
\text { for the receive side of the PMA. } \\
\text { rx_eqctrl[1:0] } \\
\text { 2'b00 Corresponding ALTGX settings } \\
\text { 2'b01 } \\
\text { 2'b10 } \\
\text { 2'b11 } \\
1 \\
2 \\
\text { 'bow) } \\
\text { (High) }\end{array}$ \\
\hline lmpi_tx_preemp & Input & $\begin{array}{l}\text { Pre-emphasis write control for the first post-tap for } \\
\text { the transmit buffer. } \\
\text { lmpi_tx_preemp[4:0] } \\
\text { settings } \\
\text { 5'b00000 }\end{array}$ \\
\hline lmpi_tx_vodctrl & Input & $\begin{array}{l}\text { Transmit buffer } \mathrm{V}_{\mathrm{OD}} \text { control signal. } \\
\text { tx_vodctrl } \\
\text { 3'bo000 }\end{array}$ \\
\hline lmpi_rx_tx_duplex_sel & Input & $\begin{array}{l}\text { Select reconfigured channel. } \\
\text { 2'b00 The transmitter and receiver portion } \\
\text { 2'b01 The receiver portion } \\
\text { 2'b10 The transmitter portion }\end{array}$ \\
\hline lmpi_write_all & Input & $\begin{array}{l}\text { Initiate a write transaction which is asserted for one } \\
\text { clock. }\end{array}$ \\
\hline lmpi_read & Input & $\begin{array}{l}\text { Read data signal, asserted for one clock. } \\
\text { Only to PMA controls reconfiguration mode. }\end{array}$ \\
\hline lmpi_channel_address & Input & $\begin{array}{l}\text { The width of this signal depends on the value you set } \\
\text { in the "What is the number of channels controlled by } \\
\text { the reconfigure controller" }\end{array}$ \\
\hline reconfig_to_xcvr & Output & $\begin{array}{l}\text { An output port of ALTGX_RECONFIG instance and } \\
\text { an input port of the ALTGX instance. }\end{array}$ \\
\hline reconfig_error & Output & Indicate that an unsupported operation is attempted. \\
\hline reconfig_busy & Output & $\begin{array}{l}\text { Indicate that the busy status of the dynamic } \\
\text { reconfiguration controller during offset cancellation } \\
\text { mode. }\end{array}$ \\
\hline xaui0_reconfig_from_xcvr & Input & An output port in the XAUI0. \\
\hline xauil reconfig from $\mathrm{xcvr}$ & Input & An output port in the XAUI1. \\
\hline
\end{tabular}

\subsection{Result analysis}

After the design of high speed XAUI based on dynamic transceiver, we did functional simulation by using VCS software from Synopsys. Then further work was done, such as synthesis, placement and routing in Quartus II software [13]. In the test bench file, we use two functions to 
record the packets information, such as simulation time, packet length, lost packet count etc.

initial begin

\$set_bus(3,2);

\$eth_init $(0,4$, eth pkt_num, \{eth_ifg,eth_bandwidth $\}, 3)$;

end

\$set_print(3,1,3,"eth_gen0.dat","eth_chk0.dat");

Check the generated file eth_gen0.dat and eth_chk0.dat. File eth gen0.dat records packets information of packets generated as the upstream input. File eth_chk0.dat details output packets of downstream, and signal xgmii_loop should be set. Table 4 shows the packets generated from $14 \mathrm{c} 76 \mathrm{~ns}$ to $15 \mathrm{cca} \mathrm{ns}$, also the packet number and the packet length are printed. Table 5 lists the received downstream packets at the output port.

Table 4. Upstream Packets Information

\begin{tabular}{|c|c|c|}
\hline TimeL & ethFCnt & length \\
\hline $14 \mathrm{c} 76$ & 86 & 588 \\
\hline $150 \mathrm{e} 3$ & 87 & 577 \\
\hline $1513 \mathrm{~d}$ & 88 & $5 \mathrm{a}$ \\
\hline $1525 \mathrm{~d}$ & 89 & 151 \\
\hline 15363 & $8 \mathrm{a}$ & $12 \mathrm{f}$ \\
\hline 15530 & $8 \mathrm{~b}$ & 230 \\
\hline $158 \mathrm{aa}$ & $8 \mathrm{c}$ & 440 \\
\hline $1593 \mathrm{~d}$ & $8 \mathrm{~d}$ & $\mathrm{a} 5$ \\
\hline $159 \mathrm{aa}$ & $8 \mathrm{e}$ & 72 \\
\hline $15 \mathrm{bf0}$ & $8 \mathrm{f}$ & $2 \mathrm{c} 1$ \\
\hline $15 \mathrm{cca}$ & 90 & $\mathrm{fb}$ \\
\hline $160 \mathrm{ea}$ & 91 & 511 \\
\hline 16563 & 92 & 582 \\
\hline $1661 \mathrm{~d}$ & 93 & $\mathrm{~d} 2$ \\
\hline
\end{tabular}

Table 5. Downstream Packet Information

\begin{tabular}{|c|c|c|}
\hline TimeL & packetNum & length \\
\hline $1666 \mathrm{a}$ & 86 & 588 \\
\hline $16 \mathrm{ad} 6$ & 87 & 577 \\
\hline $16 \mathrm{~b} 2 \mathrm{a}$ & 88 & $5 \mathrm{a}$ \\
\hline $16 \mathrm{c} 43$ & 89 & 151 \\
\hline $16 \mathrm{~d} 4 \mathrm{a}$ & $8 \mathrm{a}$ & $12 \mathrm{f}$ \\
\hline $16 \mathrm{f} 16$ & $8 \mathrm{~b}$ & 230 \\
\hline $1728 \mathrm{a}$ & $8 \mathrm{c}$ & 440 \\
\hline 17316 & $8 \mathrm{~d}$ & $\mathrm{a} 5$ \\
\hline 17383 & $8 \mathrm{e}$ & 72 \\
\hline $175 \mathrm{c} 3$ & $8 \mathrm{f}$ & $2 \mathrm{c} 1$ \\
\hline $1769 \mathrm{~d}$ & 90 & $\mathrm{fb}$ \\
\hline $17 \mathrm{ab} 6$ & 91 & 511 \\
\hline $17 \mathrm{f} 30$ & 92 & 582 \\
\hline $17 \mathrm{fe} 3$ & 93 & $\mathrm{~d} 2$ \\
\hline
\end{tabular}

These two tables illustrate that the input packets are received at the output port after a reasonable time's transmission, with the same length. In addition, eth_chk0.dat prints the lost packets count and the transmission status, so it is easy to debug.

In order to confirm the function of XAUI, we captured a screenshot on VCS simulator. Figure 5 shows the verification result of the output packets at time 15300ns, xgmii_txd[63:0] is the input signal, while xgmii_rxd[63:0] is the output signal of XAUI, and xgmii_txc[7:0] is the control byte. 


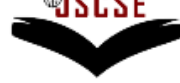

DOI: 10.7321/jscse.v2.n9.4

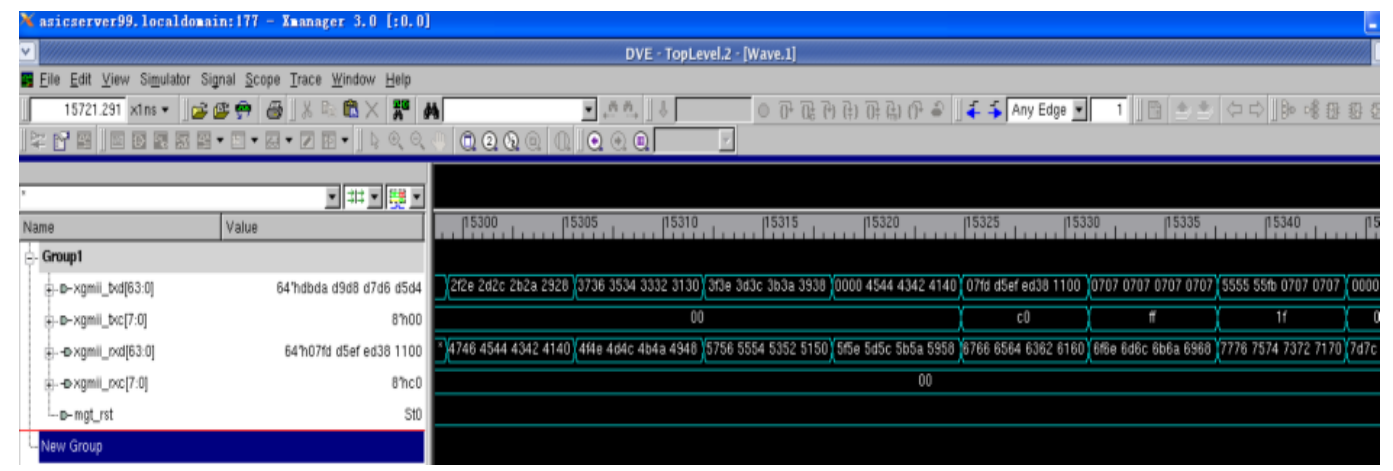

Figure 5. Transmitted and received packets at time 15300ns

When TXC is 0 , xgmii txd[63:0] present normal data transmission. When TXC is 1 , if FB byte is detected in xgmii_txd[63:0], a packet start to transmit. If FD is detected, a packet transmission is at the end.

The designed XAUI module is used on TN725 devices which developed by UTSTARCOM Co. Ltd. It is used to complete the conversion between XAUI on back panel and the internal XGMII. Though the design meets requirement quite well, it has its own limitations. First, IP cores from Altera are needed, we have to get permission from Altera. Second, timing is very important in FPGA design, meeting the timing requirements was really a hard work.

\section{Conclusions}

In the design of the proposed high speed XAUI, dynamic reconfigurable transceiver allows us to set related parameters from outside registers, which makes the best eye diagram practicable. By virtue of IP core usage, RTL coding in FPGA and SOC design becomes easy and reliable in many research areas [14]. However, there are several parameters to set, such as lmpi_tx_preemp[4:0] and Impi tx vodctrl[2:0], so it's really an arduous task which increased difficulties. In the design, two authenticated XAUI cores are adopted which work as $1+1$ protection. As a result, CPLD can generate selective signal automatically according to the position of the two PPC boards. If one of them failed to work, another will work well to keep data transmission. Though great reliability can be achieved in this method, disadvantages followed. It will reduce the channel utilization. After the process of synthesis with Quartus II 11.0, we find that 1924 LUTs (Look Up Table) of the FPGA are consumed. As a result, the design method based on IP cores is benefit to cut down design cycle, shorten press time to market, improve design efficiency and increase design reliability.

\section{References}

[1] Altera Inc., "Innovating With a Full Spectrum of 40-nm FPGAs and ASICs with Transceivers". http://www.altera.com.cn/literature/wp/wp-01078-stratix-iv-gt-40nm-transceivers.pdf

[2] Altera Inc., "Altera Devices Offer Full Support of XAUI Protocol With 10-gigabit Ethernet Reference Design". http://www.altera.com/corporate/news room/releases/releases archive/2008/products/nr-xaui.ht $\mathrm{ml}$

[3] Hou Hui, Cao Wei, Wang Jian, “Overview of Dynamic Reconfiguration”, Semiconductor Technology, vol. 33, no. 7, pp.553-557, 2008.

[4] Miao Peng, Wang Zhigong, "Research on 10 GBASE-X physical layer parallel optical transmission", Chinese High Technology Letters, vol. 17, no. 7, pp. 661-666, 2007.

[5] Zhao Lingling, Wu Zhiyong, Gao Shijie, "The Design of Single-channel Optical Transmission System Based on 10GBASE-X", Journal of Circuits and Systems, vol. 15, no. 5, pp. 112-115, 2010. 
[6] Altera Inc., “AN 558: Implementing Dynamic Reconfiguration in Arria II GX devices”. http://www.altera.com.cn/literature/hb/arria-ii-gx/an558.pdf

[7] Altera Inc., "Arria II Device Handbook Volume 2: Transceivers". http://www.altera.com.cn/literature/hb/arria-ii-gx/aiigx 5v2.pdf

[8] Chris Esser, "Xaul and InfiniBand Made Easy", Global Electronic Components, no. 9, pp. 25-26, 2002.

[9] Zhang Lei, Xia Chuanhao, "Research and Implementation of XAUI Protocol in High-speed Serial Transceiver”, CACIS·2010, pp. 88-93, 2010.

[10] Zhao Lingling, "The Design of 10Gbps Optical Transmission System Based on FPGA", Chinese Academy of Science, 2010.

[11] MA Tengfei, WU Zhiyong, LI Zeng, "10 Gb/s Optical Fiber Communication System Based on XAUI Protocol”, Computer Engineering, vol.36, no. 17, pp. 264-265,269, 2010.

[12] Xia Yuwen, Verilog Digital System Design Tutorial (The Second Edition), Press of Beijing University of Aeronautics and Astronautics, Beijing, 2008.

[13] Wu Jihua, Cai Haining, Wang Cheng. Altera FPGA/CPLD designing (Based). Posts \& Telecom Press, Beijing, 2005.

[14] Yixin Liu, Haipeng Zhang, Tao Feng, "Design of an Encryption-Decryption Module Oriented for Internet Information Security SOC Design”, International Journal of Soft Computing and Software Engineering [JSCSE], vol. 2, no. 7, pp. 26-36, 2012. 